Non-suicidal self-injury in adolescence: A longitudinal study of the relationship between NSSI, psychological distress and perceived parenting

Word count: 4850 (excluding abstract, references, tables and figures)

Imke Baetens $^{1}$, Laurence Claes ${ }^{1}$, Patrick Onghena ${ }^{1}$, Hans Grietens ${ }^{2}$, Karla Van Leeuwen ${ }^{1}$, Ciska Pieters $^{1}$, Jan R. Wiersema ${ }^{3} \&$ James W. Griffith ${ }^{1,4}$

\author{
${ }^{1}$ KU Leuven, Belgium \\ ${ }^{2}$ University of Groningen, The Netherlands \\ ${ }^{3}$ Ghent University, Belgium \\ ${ }^{4}$ Northwestern University, US
}

Correspondence concerning this article should be addressed to Imke Baetens, KU Leuven, Faculty of Psychology and Educational Science, Clinical Psychology, Tiensestraat 102, 3000 Leuven, Belgium; Phone +32.16.32.57.24, Fax +32.16.32.59.16; Email: Imke.Baetens@ppw.kuleuven.be 


\begin{abstract}
Objective: The present study investigates whether either adolescents' psychological distress and/or perceived parenting predicted the occurrence of NSSI. Furthermore, the consequences of NSSI are examined in a three-wave longitudinal study. Design: The sample at time 1 (age 12) consisted of 1396 adolescent reports and 1438 parent reports. At time 2 (age 13), 827 adolescent reports and 936 parent reports were obtained. Time 3 (age 14) included 754 adolescent reports and 790 parent reports. Psychological distress of adolescents was measured using the Strengths and Difficulties Questionnaire. Perceived parenting behaviors were examined by the Parental Behavior Scale and the Psychological Control Scale. Results: A total of $10 \%$ of the adolescents engaged in NSSI at least once before age 15 . Higher psychological distress of adolescents at time 1 was associated with the presence of NSSI at time 2 or 3 . The association between psychological distress at time 1 and perception of decreased parental rule setting at time 3 was mediated by the presence of NSSI at time 2. Conclusions: The present study showed that psychological distress at age 12 predicts NSSI over time and that parental awareness of NSSI changes the perception of parenting behaviors.
\end{abstract}

Keywords: Non-suicidal self-injury, adolescence, psychological distress, parenting, longitudinal. 


\section{Non-suicidal self-injury in adolescence: A longitudinal study of the relationship between NSSI, psychological distress and perceived parenting?}

Non-suicidal self-injury (NSSI) refers to socially unacceptable, intentional, and direct injuring of one's own body tissue without suicidal intent (Nock \& Favazza, 2009). In community samples of adolescents (aged $12-18$ ), the lifetime prevalence of NSSI was estimated to be $18 \%$ (Muehlenkamp, Claes, Havertape, \& Plener, 2012). When using a single, yes-no item, an average lifetime prevalence of $11 \%$ was reported, compared with an average lifetime prevalence of $23 \%$ when using multiple items or behavior checklists (Muehlenkamp et al., 2012). Research indicates that NSSI tends to occur first during adolescence with an average age of onset between 14-15 years (Baetens, Claes, Muehlenkamp, Grietens, \& Onghena, 2011; Heath, Toste, Nedecheva, \& Charlebois, 2009). The lifetime prevalence estimates of NSSI in young adolescents (age 10-14) have been estimated around 78\% (Hankin \& Abela, 2011; Hilt, Nock, Lloyd-Richardson, \& Prinstein, 2008), with an increase in prevalence of NSSI from 14 years onwards (Baetens et al., 2011; Hankin et al., 2011).

According to the dominant theories (e.g., Crowell, Beauchaine, \& Linehan, 2009; Nock, 2009), NSSI is related to both (1) adolescent (intrapersonal) and (2) caregivers (interpersonal) factors which shape and maintain NSSI. Several intrapersonal correlates (most importantly emotion regulation and psychological distress) are hypothesized to be interwoven with interpersonal correlates (e.g., parenting, attachment, peer influences). With regard to caregivers characteristics, parenting behaviors may play an important role in both onset of NSSI (e.g., modulating physiological arousal/regulation, invalidating emotions, stressful life events such as abuse) and in maintaining NSSI (e.g., controlling behavior of parents). Although both intrapersonal and interpersonal correlates are hypothesized as exerting influences on onset of and maintaining NSSI, until now no longitudinal studies have examined these hypotheses. Furthermore, current dominant theoretical model (e.g., Nock) lack insight into consequences of NSSI over time. This large-scale, three-wave longitudinal study examined (1) the interaction between intrapersonal and interpersonal vulnerability factors for the NSSI, and (2) the consequences of NSSI over time. The present study focuses on the interplay between psychological distress as a potential intrapersonal correlate of NSSI (i.e., levels of internalizing and externalizing 
symptoms) and perceived parenting behaviors (i.e., levels of support and control) as potential interpersonal correlates of NSSI. We examined these intra- and interpersonal pathways to NSSI at ageof-onset in a longitudinal design.

Intrapersonal correlates of NSSI

With regard to intrapersonal factors, current research shows that adolescents who self-injure report higher levels of subjective distress in response to aversive or stressful experiences (Najmi, Wegner, \& Nock, 2007; Skegg, 2005) and exhibit lower levels of distress tolerance (Anestis, Knorr, Tull, Lavender, \& Gratz, 2013; Nock \& Mendes, 2008). According Nock (2009), adolescents may exhibit NSSI to cope with elevated levels of general psychological distress, conceptualized as a wide variety of psychological symptoms (Jacobson, Muehlenkamp, Miller, \& Turner, 2008; Nock, Joiner, Gordon, Lloyd-Richardson, \& Prinstein, 2006) including both elevated rates of internalizing (e.g., anxiety, depression; e.g., Andover, Pepper, Ryabchencko, Orrico, \& Gibb, 2005) and externalizing symptoms (e.g., conduct behavioral problems; e.g., Baetens, Claes, Muehlenkamp, Grietens, \& Onghena, 2012; Brunner, Parzer, Haffner, Steen, \& Roos, 2007). In line with Baetens et al. (2012) and Klonsky and Olino (2008), it has been suggested that NSSI in community adolescents is a reflection of a general psychological distress and is not always accompanied by a diagnosable psychopathological condition. As this hypotheses has never been examined in a longitudinal perspective, the current study examines the predictive power of psychological distress on the onset and continuation of NSSI in early adolescence. The present study is the first study to examine the role of both internalizing and externalizing symptoms, conceptualized as psychological distress, in a longitudinal design. Previous research has established that internalizing symptoms (e.g., depressive symptoms) contribute to the occurrence of NSSI in two-wave longitudinal studies (Guerry \& Prinstein, 2010; Hankin \& Abela, 2011; Wilcox, Arria, Caldeira, Vincent, Pinchevsky, \& O'Grady, 2012). Furthermore, You and Leung (2012) reported that behavioral impulsivity at baseline contributed to both the occurrence and recurrence of NSSI two years later, whereas depressive symptoms failed to predict NSSI frequency. No prospective study thus far has examined the role of externalizing symptoms in predicting NSSI. Although several studies showed an association between NSSI and psychological distress (e.g., 
Running title: Longitudinal study of NSSI

Baetens et al., 2012), this association has not been investigated in a longitudinal design.

\section{Interpersonal correlates of NSSI}

Parenting behavior is considered the observable behavior of a parent in interaction with his/her child (Rollins \& Thomas, 1979). As reviewed by Rollins and Thomas (1979), parenting behaviors can be placed upon a continuum of support (behavior wherein a parent shows warmth, acceptation and understanding to the child) and a continuum of control (behavior wherein a parent wishes to influence the behavior of the child). On the latter dimension, behaviors are classified into parental control of the child's psychological world (psychological control) and control of the child's behavior by punishment, including harsh punishment (behavioral control; Barber et al., 2005). Parenting behavior dimensions can be combined into parenting styles. Combining the two dimensions support and control, four main parenting styles can be distinguished: indulgent (low control, high support), authoritarian (high control, low support), authoritative (high control, high support) and uninvolved (low control, low support) parenting (e.g., Barber, 1996; Baumrind, 1991).

Several studies (e.g., Barber et al., 2005; Berg-Nielsen, Vikan, \& Dahl, 2002) have shown that high parental support and low parental control are associated with higher levels of adaptive psychosocial functioning (e.g., competence, self-regulation, and academic achievement) and lower levels of internalizing and externalizing problem behaviors. In relation to NSSI behaviors, Bureau et al. (2010) found a positive association between NSSI and perceived parental control, and a negative association between NSSI and perceived support. Previous research (e.g., Baetens et al., 2013; Martin \& Waite, 1994; Patton, Coffey, Posterino, Carlin, \& Wolfe, 2001) has identified an authoritarian parenting style, conceptualized as high behavioral control and low support, to be a significant risk factor for NSSI, depression, suicide attempts as well as completed suicide during adolescence. Nonetheless, no study thus far has examined parenting behaviors, specifically the combination of low supportive and high controlling parenting behaviors, as a vulnerability for engaging in NSSI in adolescence in a longitudinal design.

Causality between perceived parenting and NSSI may go in both directions. Negative parenting practices can increase the probability of NSSI, but the reverse can also be present - negative 
parenting can also be a consequence of NSSI. In parenting studies, results have shown that adolescents' problem behaviors affect the perceived family environment, rather than vice versa. For example, Huh, Tristan, Wade, and Stice (2006) found that adolescent girls' problem behavior had a greater impact on parenting than parenting did on the adolescent girls' problem behavior. Similarly, Hafen and Laursen (2009) reported that initial levels of adolescents' externalizing symptoms predicted subsequent changes in perceived parental support. With regard to adolescents' problem behavior and perceived family relationships, Lubenko and Sebre (2010) found that adolescent factors affected family relationship quality rather than vice-versa: Elevations in adolescents' problem behavior prospectively predicted a change in perceived family relationships. These studies suggest the necessity to examine adolescents' problem behavior in relation to perceived parenting in a longitudinal design.

Only one study thus far examined the effect of NSSI on the family and found support for a social positive reinforcement mechanism (Hilt et al., 2008): Individuals seemed to engage in NSSI in order to obtain more social support. Results showed an increase in positive relationship quality in the father-daughter relationship from time 1 to time 2, due to NSSI. No studies thus far have examined whether differences in perceived parenting are related to parental awareness of NSSI behaviors.

Aims of the study

This prospective longitudinal study examines intra- and interpersonal factors as antecedents and consequences of NSSI behaviors.

The first aim of the present study was to investigate vulnerability factors for engaging in NSSI. We examined both intrapersonal correlates (psychological distress) and interpersonal correlates (perceived parenting behaviors). Specifically, we aimed to investigate whether either psychological distress and/or perceived parenting predicted the occurrence of NSSI. Psychological distress and perceived parenting were both hypothesized to predict NSSI. Consistent with Martin and Waite (1994), we hypothesized a significant interaction between perceived parental support and parental control. More specifically, we expected low parental support in combination with high parental control to be a risk factor for NSSI over time.

Several studies have demonstrated gender differences in NSSI correlates, psychological 
symptoms and perceived parenting behaviors. Moreover, differences have been found between community samples and clinical samples in NSSI correlates. Thus, both gender and diagnosis of psychiatric disorder were entered in our models as covariates.

The second aim was to examine the effects of NSSI on parenting behaviors. We expected to find differences in perceived parenting behaviors as a result of NSSI behaviors. We hypothesized that psychological distress would predict NSSI behaviors, which would in turn cause a difference in perception of parenting behavior. In order to test this hypothesis, mediation analyses were performed using individual parenting constructs (positive parenting, parental rule setting, harsh punishment and psychological control). Consistent with Hilt et al. (2008), we hypothesized a social positive reinforcement mechanism whereby NSSI is exhibited as a means to obtain more social support (i.e., more positive parenting). Finally, because NSSI is often a secretive behavior, we examined whether differences in perceived parenting were related to parents being aware of NSSI or not.

\section{Method}

\section{Procedure and participants}

Participants were drawn from the JOnG! study (Grietens, Hoppenbrouwers, Desoete, Wiersema, \& Van Leeuwen, 2010), a prospective cohort study of Flemish adolescents aged twelve to fifteen, as well as their parents. The sample was recruited from eight districts in Flanders including both urban and rural areas. The key objectives of JOnG! were to follow the development of mental health, family relationships, and healthcare from pre-adolescence until adolescence.

In March 2009, all pre-adolescents (age 12) and parents of twelve-year old adolescents living in eight districts of Flanders $(N=9861)$ were invited to participate in this study. This is $14 \%$ of all twelve-year olds in Flanders (Grietens et al., 2010). All participants, both adolescents and parents, gave informed consent for their reports to be used. In addition, a parent or legal guardian provided informed consent for the data of adolescent-report. The study procedures were approved by the Ethics Committee of both universities cooperating in the JOnG! project.

At time 1, 1443 twelve-year old adolescents participated in this study with a response rate of $14.63 \%$ (1443/9861). The sample at time 1 consisted of 1397 valid $(96.74 \%$; i.e., less than $10 \%$ 
missing data) adolescent reports at age 12 , of whom $54.70 \%$ girls and $45.30 \%$ boys. At time 1,1438 valid parent reports were administered of whom $88.70 \%$ mothers, $4.30 \%$ fathers, $1.2 \%$ step, adoptive or foster parents. At age 13 (time 2), 1132 adolescents and their parents participated in this study resulting in 827 valid adolescent reports and 936 parent reports. At ages 14-15 (one and a half years after time 2), 839 adolescents and their parents participated, resulting in 754 valid adolescent reports and 790 valid parent reports.

Data were missing at different time points for different participants. Using Little's (1988) Missing Completely At Random (MCAR) test, participants with and without complete data were compared in terms of gender, district, presence of psychiatric disorder, and psychological complaints (SDQ). The MCAR test resulted in a non-significant Chi-square value, $\chi^{2}(177)=175.70, n s$, rejecting the null hypotheses that the missing data at different time points are not MCAR.

Data analyses were conducted with Mplus. Mplus provides maximum likelihood estimates of model parameters under the assumptions that data are missing completely at random or missing at random (Muthén \& Muthén, 2010). For logistic regression, the maximum likelihood estimator with robust standard errors was used (MLR). For mediation analyses, a robust weighted least squares estimator (WLSMV) was used, with probit links for categorical dependent variables.

\section{Measures}

Engagement in NSSI was determined by an affirmative response to the item 'Have you intentionally injured yourself (e.g., cut, burn, scratch) since the previous survey, without the intent to die?'. At age 12, the lifetime prevalence of NSSI was questioned using 'Have you ever injured yourself, without the intent to die?'. Whether parents were aware of NSSI of their sons or daughters was questioned as follows: 'Has your son/daughter ever intentionally injured him/herself - for example, cutting, burning, scratching - without the intent to die?'. The NSSI-screening questions were dummy-coded with " $0=$ no" and " 1 = yes". Using a single-item measure of NSSI is common in NSSI research and has been shown to render consistent estimates of prevalence (Muehlenkamp et al., 2012).

Parents of respondents were asked whether their sons or daughter ever received a formal diagnosis of an emotional, behavioral or developmental disorder (e.g., depression, anxiety, mental 
retardation, autism, ADHD et cetera). The presence of any psychiatric disorder was dummy-coded with " $0=$ no disorder" and " $1=$ at least one disorder present".

Psychological complaints of the adolescents were examined by adolescent-reported Strengths and Difficulties Questionnaire (SDQ; Goodman, 2001). The SDQ is a well-known, brief screening questionnaire, which includes the following scales: emotional symptoms, conduct problems, hyperactivity/inattention, peer relationship problems and pro-social behaviors. The impact score and the pro-social behaviors subscales were not used in the present study.

Parenting behavior was measured using the adolescent-reported Parental Behavior Scale, shortened version (PBS; Van Leeuwen \& Vermulst, 2004). Only the subscales of positive parenting, parental rule setting, punishing, and harsh punishing were used in the longitudinal analyses. The first two subscales, positive parenting and parental rules-setting, were used as indicators of the parenting concept of support. In this measurement, parental rule setting is formulated in terms of both learning rules and setting limits. The subscales for punishment and harsh punishment were used as indicators of negative control. Psychological control was measured using the Psychological Control Scale (PCS; Barber, 1996). Adolescents were asked to clarify which parent/legal guardian they had in their mind when answering questions regarding parenting behaviors. More than $90 \%$ (at all three time points) reported that they answered the PBS with regard to parenting behaviors of their mother.

Prior to conducting regression analyses, a confirmatory factor analysis was used to examine the factors for 'psychological distress' and 'perceived parenting behaviors'. The latent factor "psychological distress" is a reflection of an amount of psychological symptoms, containing four subscales of the adolescent-report Strengths and Difficulties Questionnaire (SDQ). The higher-order factor "perceived parenting behaviors" contained two indicators: positive parenting and controlling behaviors. Positive parenting contained two indicators: positive behaviors and parental rules setting. Controlling behaviors contained the two PBS subscales for punishment and harsh punishment and psychological controlling behaviors measured by eight items of the Psychological Control Scales (PCS). The factor loadings were set to be equal in order to identify the model. For the confirmatory factor analysis, we evaluated model fit using three goodness-of-fit indices: the comparative fit index 
(CFI; Bentler, 1990; Hu \& Bentler, 1999), the Tucker-Lewis Index (TLI; McDonald \& Marsh, 1990; Tucker \& Lewis, 1973), and the root mean square error of approximation (RMSEA; Browne \& Cudeck, 1993). We used the following fit indices: CFI and RMSEA are above the cut point recommended $(>.90$ and $<.08$, respectably), and TLI $(>.95)$, as suggested by Brown (2006). Although we used cut-off values as a guide to model interpretation, we did not necessarily reject models if certain fit indices were slightly outside these cut-off values (for a discussion, see Marsh, Hau, \& Wen, 2004).

The indicators for global adjustment of the first model with the nine subscales of the three instruments (SDQ, PBS and PCS) were lower than the acceptable level $\left[\chi^{2}(27)=290.82\right.$, $\mathrm{p}<.001 / \mathrm{TLI}=.79 / \mathrm{CFI}=.84 / \mathrm{RMSEA}=.10]$. By examining the indicators for local adjustment it was possible to identify the subscale PBS punishment that had low values of $R^{2}(0.18 \pm 0.01)$, which indicates small amount of variance explained by the proposed model (Kline, 2005). Therefore the subscale PBS punishment was removed, resulting in a model composed of three latent variables (psychological distress, perceived positive parenting and perceived controlling behavior), which we hypothesized to have four indicators for the first factor, two for the second factor, and two for the third factor. This option was based on the assumption of improving the adjustment and validity of the model and the PBS subscale punishment was not considered essential for the construct perceived controlling behaviors.

Our CFA model is presented in Figure 1. The model fit was evaluated based on three goodness-of-fit indices. We considered the model to have an acceptable fit, $\chi^{2}(19)=170.81, p<.001$, $T L I=.85, C F I=.90, R M S E A=.09]$, although the TLI and RMSEA are slightly outside the recommended cutoffs $(>.95$ and $<.08$, respectively).

Analyses

Data were analyzed using SPSS and Mplus (Muthén \& Muthén, 2010). The internal consistency of each of the subscales was assessed using Cronbach's alpha. Table 1 presents means, standard deviations and Cronbach's alphas for all relevant scales. To investigate the associations between scales, the Pearson correlation coefficient was used. To determine the associations between 
the scales and the dichotomous NSSI item, point-biserial correlations were used.

For all continuous subscales, we calculated Percent of Maximum Possible Scores (POMP: Cohen, Cohen, Aiken, \& West, 1999). POMP scores rescale questionnaires such that the score is the percentage of the distance $(0-100 \%)$ from the minimum to the maximum of a scale. POMP scores allow us to examine both the magnitude and impact of the observed relationships between variables even when the underlying units of metric are different.

Logistic regression analyses, using sets of variables based on theory, were used to analyze the predictive power of psychological distress (PS) and perceived parenting behaviors (PP \& CP) in relation to NSSI behaviors at time 2 or time 3 . NSSI at time 1 , gender $(0=$ male; $1=$ female $)$ and the presence of psychiatric disorder (0/1) were entered in step 1. Step 2 included hypothesized predictors: psychological distress (PS), positive parenting (PP), and controlling parenting behaviors (CP). Step 3 included the interaction between positive parenting (support) and controlling parenting behaviors (control).

We also tested mediation using a bootstrapping method (Preacher \& Hayes, 2008). We used 1000 resamples to derive a $95 \%$ confidence interval for the indirect effect of the independent variable via the mediator to the dependent variable.

\section{Results}

At time 1, 72 adolescents (out of 1397 twelve-year olds) indicated that they injured themselves on purpose, without suicidal intent, giving a prevalence of NSSI at twelve years of slightly more than $5 \%$. No significant gender difference was found: $44.44 \%$ of the NSSI group were boys, and $55.66 \%$ were girls $\left.\left(\chi^{2}(1), N=1397\right)=.07 ; p=.79\right)$.

At time 2, 23 out of 827 thirteen-year olds reported NSSI the past year for a one-year prevalence estimate of $2.78 \%$ at age 13 , with no significant difference across gender $\left(\chi^{2}(1), N=827\right)=$ $2.70 ; p=.10)$. Out of 921 parents who answered on the NSSI screening question at time $2,1.52 \%$ (14/921) of the parents reported a lifetime prevalence of NSSI of their sons/daughters.

At time 3, 5.31\% (40/754) of the respondents reported NSSI behavior at least once in the past year. At time 3, a significant gender difference was found, with girls being 3 times more likely to 
report NSSI behaviors $\left.\left(\chi^{2}(1), N=752\right)=5.33 ; p=.02\right)$. Out of 786 parent reports on the NSSI screening question at time $3,3.18 \%(25 / 786)$ of the parents reported a lifetime prevalence of their son/daughter.

Out of 533 valid adolescent reports participating in all three time points, $10.70 \%(57 / 533)$ reported having engaged in NSSI at least once, only a minority $(0.9 \% ; 5 / 57)$ reported having engaged in NSSI in at least two time points.

Aim 1: Psychological distress and perceived parenting as predictors for NSSI on time 2 and time 3 (statistically controlling for NSSI at time 1, gender and psychiatric diagnoses)

We conducted a regression analysis to investigate the unique predictive power of both psychological distress at time 1 and perceived parenting behaviors (both positive parenting and controlling behaviors) at time 1 using the presence versus absence of NSSI at time 2 or 3 as the dependent variable.

NSSI at time 1, gender and psychiatric diagnoses at time 1 were entered in Step 1 of a regression using NSSI at time 2 or 3 as the dependent variable. The latent factors psychological distress (containing emotional problems, conduct problems, hyperactivity, and peer problems), positive parenting (positive support and parental rules setting) and controlling behavior (harsh punishment, psychological control) were entered in Step 2. The interaction between positive parenting and controlling behavior were entered in step 3 .

Gender predicted NSSI (present at time 2 or 3) with boys being less likely to engage in NSSI. Higher psychological distress was associated with presence of NSSI at time 2 or $3(O R=2.26)$, even after statistically controlling for NSSI at time 1, gender and psychiatric disorder at time 1 (Table 2). The results show no unique significant predictive power for perceived positive parenting and controlling parental behavior, nor for the interaction between perceived positive parenting and controlling behavior.

\section{Aim 2: Differences in perceived parenting behaviors due to engaging in NSSI behaviors}

Having established an association between psychological distress at time 1 and perceived parenting behaviors at time 3 (PS1-PP3 $r=-.23^{* * *}$; PS1-CP3 $\left.r=.26^{* * *}\right)$, we tested whether this 
relationship was mediated by NSSI at time 2 . In order to enlarge insight into the specific associations between NSSI and perceived parenting, Table 3 shows the regression coefficients for the mediation model in the prediction of the individual perceived parenting constructs.

Psychological distress was significantly correlated with a perception of an increased amount of parental rules setting at time 3 , though this effect was mediated by the presence of NSSI. Adolescents who have a higher level of psychological distress are more likely to engage in NSSI. Engaging in NSSI in turn, leads to a lower amount of perceived parental rules setting.

Mediation analyses with other perceived parenting behaviors such as positive parenting, harsh punishment and psychological control were not significant (see table 3).

Aim 3: Impact of parental awareness of NSSI

Whether parental awareness of NSSI is relevant to the change in perception of parenting behaviors (i.e., parental rule setting) was examined using mediation analyses (Figure 3). No significant indirect effect of parents knowing about NSSI was found, but a direct effect of elevated psychological distress on parental awareness, as well as a direct effect between parental awareness of NSSI and an increased perception of parental rules setting were found. Increased perception of parental rules setting is therefore associated with both increased psychological distress and parental awareness of NSSI, without parental awareness of NSSI behavior being a significant mediator.

\section{Discussion}

The present study is the first prospective three-wave investigation of NSSI in early adolescence. The goal of this study was to examine the frequency of NSSI behaviors in a large community sample of adolescents. The NSSI prevalence rates found are slightly higher than prevalence rates in young adolescence in previous studies (Hankin et al., 2011; Hilt et al., 2008), with a total prevalence of $10.70 \%$ at three time points. The results are supportive with prevalence rates found in Flanders (Baetens et al., 2011). In line with Hankin et al. (2011), the yearly prevalence of NSSI starts increasing at age 14 , although $5 \%$ of the pre-adolescents already engaged in NSSI at age 12. The increasing incidence of NSSI from age 14 onwards can be understood as arising from the interplay between aberrations of maturational neurobiological changes (Paus, Keshavan, \& Giedd, 
2008) and increasing transition into adulthood (and related decreasing independence upon caregivers) that typically occur in adolescence.

In clinical samples of adolescents, NSSI is highly prevalent in females. Results from nonclinical samples suggest that males and females may not differ with respect to rates of NSSI, but may differ in the form of self-injury. Heath et al. (2009) concluded that when research includes only NSSI acts (cutting, burning, self-hitting and other forms of tissue damage) without other forms of selfharming behaviors, no significant gender differences are found. Types of self-injury appear to differ by gender, with boys being more likely to hit or burn whereas girls, are more likely to cut (Baetens, Claes, Willem, Muehlenkamp, \& Bijttebier, 2011). The current study shows that boys are less likely to report NSSI at follow-up, consistent with results of Whitlock et al. (2011) in a young-adult sample.

Psychological distress, rather than only internalizing symptoms as reported by several studies (e.g., Guerry et al., 2010; Hankin et al., 2011; Wilcox et al., 2012), is a significant predictor of NSSI, even after statistically controlling for NSSI at time 1, gender, and psychiatric disorder at time 1 . However, further research on the role of externalizing symptoms, such as hyperactivity, frustration and aggression, is needed. As pointed out by Baetens et al. (2012), NSSI is a result of heightened psychological distress that is common during the course of adolescent development, rather than a result of diagnosable psychopathology. When taking into account adolescent psychological distress, perceived parental behaviors are no longer significant predictors of NSSI behavior.

Most NSSI research examines correlates of NSSI, but there is a lack of longitudinal research on the antecedents and consequences of NSSI. In line with the theories of Crowell et al. (2009) and Nock (2009), results of the current study show that psychological distress is a significant predictor of NSSI over time, leaving less variance to perceived parenting on the onset of NSSI. Nonetheless, results suggest an underlying effect of perceived parenting in continuation of NSSI. The mere presence of NSSI seems to have an effect on the perception of parenting behaviors over time:

Adolescents who self-injure tend to perceive a decrease in parental rule setting over time. This can be understood in the light of the social interaction theory (Coyne, 1976): parents tend to decrease limit setting, and increase of autonomy and parental trust and support when being worried about the well- 
being of their child parents. But when parents are aware of NSSI behaviors, adolescents report more parental rule setting. These results can be understood in the light of the social cognitive theory (Bandura, 1986). When confronted with NSSI of sons/daughter, parents may increase limit setting as means to get grip at the situation (and their own fears and worries). This may indicate a mere interpretation by the adolescent, but can also point to a real increase in limit setting by parents as a means to control the behavior of their self-injurious child. To get insight into the consequences and effects of NSSI on the family over time, future research should examine perception of parents and siblings via multi-wave and multi-informant designs.

The present study explored the interplay between intrapersonal (psychological distress) and interpersonal risk factors (perceived parenting behaviors) in relation to each other in the prediction of NSSI behaviors in adolescence. We used a prospective, multi-wave assessment of NSSI with yearly follow-ups to reduce recall problems and to improve accuracy.

The study has also limitations that need to be addressed in future research. First, we relied on adolescent-report to measure psychological distress and perceived parenting behaviors. Highly psychologically distressed adolescents might have a negative cognitive bias regarding their perception of their environment (e.g., due to depressive symptoms; Beck, 1987), so future research should examine parent-reported differences in parenting behaviors as antecedents and consequences. Second, we used a broad parent-reported screening for psychiatric disorders. Future research could benefit from formal diagnostic interviews. Third, although the response rate to the written questionnaire was only $15 \%$, the sample size was still large $(N=1443)$. It was previously shown to be a fair representation of the Flemish adolescents in this age group, with respect to ethnic origin and multiple indicators of socioeconomic status (i.e., educational level and employment of parents, and family income) (Guérin et al., 2012). Fourth, the present study was conducted in a community sample, so the results might not generalize to clinical populations. Future research should test these results in clinical samples. Fifth, more than $90 \%$ of the adolescents reported perceived parenting by their mother. Due to a small amount of perceived parenting behaviors by father, we fail to compare perceived parenting by mother and father. Hilt et al. (2008) only found a positive reinforcement mechanism with regard to quality of 
relationship with father. Future research would benefit from examining parental behavior by mothers and fathers separately. Finally, in our regressions, we included NSSI at time 1 as a covariate, as well as gender and psychiatric disorder. This perhaps left less variance to be explained by the hypothesized predictors. To examine more complex models of the interplay between interpersonal and intrapersonal correlates of NSSI, future research should target adolescents engaging in NSSI. Research in community samples, for example, of adolescents with depressed mothers (Hankin et al., 2011) would increase the likelihood that NSSI would be present and change over time. Larger groups of adolescents engaging in NSSI would increase statistical power and permit the investigation of more covariates and more complex models.

In sum, the present study found that psychological distress at age 12 predicted NSSI over time. There was also a significant effect of gender with boys being less likely than girls to report NSSI behaviors at follow-up. Mediation analyses supported that NSSI has an effect on the perception of parenting behaviors, in the sense that adolescents report lower levels of parental rule setting. However when parents are aware of NSSI, adolescents perceive an increase of parental rule setting. The present study suggests that the process by which intrapersonal and interpersonal risk factors are associated with NSSI is complex and more research is needed on the bidirectional effects of NSSI and its risk factors.

\section{Implications for practice, application, and policy}

Results of the present study show that approximately one in three parents are aware that their son/daughter are engaging in NSSI. Only $50 \%$ of adolescents who self-injure disclose this behavior to anyone else (Baetens et al., 2011), and in most cases the persons who are aware of their self-injury are peers (Evans, Rodham, \& Hawton, 2005). Considering that up to $70 \%$ of those adolescents engaging in NSSI are likely to attempt suicide at least once (Muehlenkamp, Williams, Gutierrez, \& Claes, 2009), the need for social awareness of NSSI at a societal level is vital. Providing information to the public, in particular adolescents from age 12-18, and stimulating a public debate might rupture the taboo of NSSI and break the cycle of isolation for adolescents engaging in NSSI.

The present findings present meaningful ideas for intervention and prevention of NSSI in 
Running title: Longitudinal study of NSSI

community samples. Psychological distress is found to be a significant predictor of NSSI over time, suggesting a benefit of large-scale prevention programs targeting general well-being of young adolescents. For example, 'learning adaptive coping' in the school's curriculum might reduce mild forms of NSSI. In treatment it is important to involve family members, to counter the effect of NSSI on the family. 


\section{References}

Andover, M.S., Pepper, C.M., Ryabchencko, K.A., Orrico, E.G., \& Gibb, B.E. (2005). Self-mutilation and symptoms of depression, anxiety, and borderline personality disorder. Suicide and LifeThreatening Behavior, 35, 581-591. doi: 10.1080/13811110903479086.

Baetens, I., Claes, L., Muehlenkamp, J.J., Grietens, H., \& Onghena, P. (2011). Non-suicidal and suicidal self-injurious behavior among Flemish adolescents: A websurvey. Archives of Suicide Research, 15, 56-67. doi: 10.1080/13811118.2011.540467.

Baetens, I., Claes, L., Muehlenkamp, J.J., Grietens, H., \& Onghena, P. (2012). Differences in psychological symptoms and self-competencies in non-suicidal self-injurious Flemish adolescents. Journal of Adolescence, 35, 753-759. doi:10.1016/j.adolescence.2011.11. 001.

Baetens, I., Claes, L., Onghena, P., Grietens, H., Van Leeuwen, K., Pieters, C., Wiersema, J.R., \& Martin, G. (2013). Is non-suicidal self-injury associated with parenting and family factors? Journal of Early Adolescence. doi: 10.1177/0272431613494006

Baetens, I., Claes, L., Willem, L., Muehlenkamp, J.J., \& Bijttebier, P. (2011). The relationship between non-suicidal self-injury and temperament in male and female adolescents based on child- and parent-report. Personality and Individual Differences, 50, 527-530. doi: 10.1016/j.paid.2010.11.015.

Bandura, A. (1986). Social foundations of thought and action: A social cognitive theory. Englewood Cliffs, NJ: Prentice-Hall.

Barber, B.K. (1996). Parental psychological control: Revisiting a neglected construct. Child Development, 67, 3296-3319. doi: 0009-3920/96/6706-0034\$01.00.

Barber, B.K., Stolz, H. E., \& Olsen, J.A. (2005). Parental support, psychological control, and behavioral control: Assessing relevance across time, culture, and method. Monographs of the Society for Research in Child Development, 70, 1-137. doi: 10.1111/j.15405834.2005.00365.x

Barrocas, A. L., Jenness, J. L., Davis, T. S., Oppenheimer, C. W., Technow, J. R., Gulley, L. D., ... \& Hankin, B. L. (2010). Developmental perspectives on vulnerability to nonsuicidal self-injury 
Running title: Longitudinal study of NSSI

in youth. Advances in child development and behavior, 40, 301-336.

Barrocas, A. L., Jenness, J. L., Davis, T. S., Oppenheimer, C. W., Technow, J. R., Gulley, L. D., ... \& Hankin, B. L. (2010). Developmental perspectives on vulnerability to nonsuicidal self-injury in youth. Advances in child development and behavior, 40, 301-336.

Baumrind, D. (1991). Parenting styles and adolescent development. In Lerner, R.M., Petersen, A. S., \& Brooks-Gunn, J. (Eds.), Encyclopedia of Adolescence (pp. 758-772). New York: Garland Publications.

Beck, A.T. (1987). Cognitive models of depression. Journal of Cognitive Psychotherapy, 1, 5-37.

Bentler, P.M. (1990). Comparative fix indexes in structural models. Psychological Bulletin, 107, 238246. doi: 10.1037/0033-2909.107.2.238.

Berg-Nielsen, T.S., Vikan, A., \& Dahl, A.A. (2002). Parenting related to child and parental psychopathology: A descriptive review of the literature. Clinical Child Psychology and Psychiatry, 7, 529-552. doi: 10.1177/1359104502007004006.

Brown, T. (2006). Confirmatory factor analysis for applied research. New York: The Guilford Press.

Browne, M.W., \& Cudeck, R. (1993). Alternative ways of assessing model fit. In Bollen, K. A. \& Long, J. S. (Eds.), Testing structural equation models (pp. 136-162). Beverly Hills, CA: Sage.

Brunner, R., Parzer, P., Haffner, J., Steen, R., \& Roos, J. (2007). Prevalence and psychological correlates of occasional and repetitive deliberate self-harm in adolescents. Archives Pediatric Adolescence, 161, 641-649. doi:10.1001/ archpedi.161.7.641.

Bureau, J.F., Martin, J., Freynet, N., Poirier, A.A., Lafontaine, M.F., \& Cloutier, P. (2010). Perceived dimensions of parenting and non-suicidal self-injury in young adults. Journal of Youth and Adolescence, 39, 484-494. doi: 10.1007/s10964-009-9470-4.

Chapman, A. L., Gratz, K. L., \& Brown, M. Z. (2006). Solving the puzzle of deliberate self-harm: The experiential avoidance model. Behavior Research and Therapy, 44, 371-394. doi:10.1016/j.brat.2005.03.005.

Cohen, P., Cohen, J., Aiken, L., \& West, S. (1999). The problem of units and the circumstance for POMP. Multivariate Behavioral Research, 34, 315-246. 
Running title: Longitudinal study of NSSI

Coyne, J.C. (1976). Depression and the response to others. Journal of Abnormal Psychology, 85, 186193. doi: $10.1037 / 0021-843 X .85 .2 .186$

Crowell, S. E., Beauchaine, T. P., \& Linehan, M. M. (2009). A biosocial developmental model of borderline personality: Elaborating and extending Linehan's theory. Psychological bulletin, 135, 495-510. doi: 10.1037/70015616.

Crowell, S. E., Beauchaine, T. P., \& Linehan, M. M. (2009). A biosocial developmental model of borderline personality: Elaborating and extending Linehan's theory. Psychological bulletin, 135, 495-510. doi: 10.1037/70015616.

Evans, E., Hawton, K., \& Rodham, K. (2005). In what way are adolescents who engage in self-harm or experience thoughts of self-harm different in terms of help-seeking, communication and coping strategies? Journal of Adolescence, 28, 573-578. doi: 10.1016/j.adolescence.2004.11.001.

Goodman, R. (2001). Psychometric properties of the Strengths and Difficulties Questionnaire (SDQ). Journal of the American Academy of Child and Adolescent Psychiatry, 40, 13371345. doi:10.1097/00004583-200111000-00015.

Gratz, K. L. (2007). Targeting emotion dysregulation in the treatment of self-injury. Journal of Clinical Psychology, 63, 1091-1103. doi:10.1002/jclp.20417.

Grietens, H., Hoppenbrouwers, K., Desoete, A., Wiersema, J.R., \& Van Leeuwen, K. (2010). JOnG! theoretische achtergronden, onderzoeksopzet en verloop van het eerste meetmoment. [JOnG! theoretical background, research design and methodology of the first wave]. Policy Research Centre of Welfare, Health and Family: Leuven. Retrieved from: http://steunpuntwvg.be/20072011/swvg/_docs/Publicaties/2010JONG_Hans\%20Grietens_zonder\%20bijlagen_website.pdf

Guérin, C., Pieters, C., Roelants, M., Van Leeuwen, K., Desoete, A., Wiersema, J.R., \& Hoppenbrouwers, K. (2012). Socio-demografisch profiel en gezondheid van 6 en 12-jarige jongeren (cohorte JOnG!) in Vlaanderen. [Sociodemographic profile and health of 6- and 12years-old youngsters (cohorts JOnG!) in Flanders]. Policy Research Centre of Welfare, Health and Family, Leuven. Retrieved from: www.steunpuntwvg.be. 
Running title: Longitudinal study of NSSI

Hafen, C., \& Laursen, B. (2009). More problems and less support: Early adolescent adjustment forecasts changes in perceived support from parents. Journal of Family Psychology, 23, 193202. doi: $10.1037 / \mathrm{a} 0015077$.

Hankin, B.L., \& Abela, J.R.Z. (2011). Nonsuicidal self-injury in adolescence: Prospective rates and risk factors in a $2 \frac{1}{2}$ year longitudinal study. Psychiatry Research, 186, 65-70. doi:10.1016/j.psychres.2010.07.056.

Heath, N.L., Schaub, K.M., Holly, S., \& Nixon, M.K. (2009). Self-injury today: Review of population and clinical studies in adolescents. In M. K. Nixon \& N. L. Heath (Eds.), Self-Injury in Youth: The Essential Guide to Assessment and Intervention (pp. 9-28). New York, N.Y.: Routledge Press.

Hilt, L.M., Nock, M.K., Lloyd-Richardson, E.E., \& Prinstein, M.J. (2008). Longitudinal study of nonsuicidal self-injury among young adolescents: Rates, correlates and preliminary tests of an interpersonal model. Journal of Early Adolescence, 28, 455-469. doi: 10.1177/0272431608316604.

Hu, L., \& Bentler, P.M. (1999). Cutoff criteria for fit indices in covariance structure analysis: Conventional criteria versus new alternatives. Structural Equation Modeling, 6, 1-55. doi: 10.1080/10705519909540118.

Huh, D., Tristan, J., Wade, E., \& Stice, E. (2006). Does problem behavior elicit poor parenting? A prospective study of adolescent girls. Journal of Adolescent Research, 21, 185-204. doi: $10.1177 / 0743558405285462$.

Jacobson, C.M., Muehlenkamp, J.J., Miller, A.L., \& Turner, J.B. (2008). Psychiatric impairment among adolescents engaging in different types of deliberate self-harm. Journal of Clinical Child and Adolescent Psychology, 37, 363-375. doi: 10.1080/15374410801955771.

Kline, R. (2005). Principles and practice of structural equation modeling (2th ed.). New York: The Guilford Press.

Klonsky, E. D., \& Muehlenkamp, J. J. (2007). Self-injury: A research review for the practitioner. Journal of Clinical Psychology, 63, 1045-1056. doi:10.1002/jclp.20412. 
Running title: Longitudinal study of NSSI

Little, R.J.A. (1988). A test of missing completely at random for multivariate data with missing values. Journal of the American Statistical Association, 83 (404), 1198-1202.

Lubenko, J., \& Sebre, S. (2010). Longitudinal associations between adolescent behavior problems and perceived family relationships. Procedia Social and Behavioral Sciences, 5, 785-790. doi: 10.1016:j.sbspro.2010.07.185.

Marsh, W.H., Hau, K., \& Wen, Z. (2004). In search of golden rules: Comment on hypothesis-testing approaches to setting cutoff values for fit indexes and dangers in overgeneralizing $\mathrm{Hu}$ and Bentler's (1999) findings. Structural Equation modeling, 11, 320-341.

Martin, G., \& Waite, S. (1994). Parental bonding and vulnerability to adolescent suicide. Acta Psychiatrica Scandinavica, 89, 246-254. doi: 10.1111/j.1600-0447.1994.tb01509.x.

McDonald, R.P., \& Marsh, H.W. (1990). Choosing a multivariate model: Noncentrality and goodness of fit. Psychological Bulletin, 107, 247-255.

Muehlenkamp, J.J., Claes, L., Havertape, L., \& Plener, P. (2012). International prevalence of adolescent non-suicidal self-injury and deliberate self-harm. Child and Adolescent Psychiatry and Mental Health, 6: 10. doi:10.1186/1753-2000-6-10. Retrieved from the WWW on March the $20^{\text {th }}$ of 2013 on http://www.capmh.com/content/6/1/10.

Muehlenkamp, J.J., Williams, K.L., Gutierrez, P.M., \& Claes, L. (2009). Rates of non-suicidal selfinjury in high school students across five years. Archives of Suicide Research, 13, 317-329. DOI: $10.1080 / 13811110903266368$.

Muthén, L.K., \& Muthén, B. O. (2010). Mplus user's guide (6th ed.). Los Angeles, CA: Muthén \& Muthén.

Najmi, S., Wegner, D. M., \& Nock, M. K. (2007). Thought suppression and self-injurious thoughts and behaviors. Behavior Research \& Therapy, 45, 1957-1965. doi:10.1016/j.brat.2006.09.014.

Nock, M. K. (2009).Why do people hurt themselves? New insights into the nature and functions of self-injury. Current Directions in Psychological Science, 18, 78-83. doi:10.1111/j.14678721.2009.01613.x.

Nock, M., \& Favazza, A. (2009). Nonsuicidal self-injury: definition and classification. In M. Nock 
Running title: Longitudinal study of NSSI

(Ed.) (2009), Understanding non-suicidal self-injury: Origins, assessment and treatment (pp.

9-18). Washington, DC: American Psychological Association.

Nock, M.K., \& Mendes, W.B. (2008). Psychological arousal, distress tolerance, and social problemsolving deficits among adolescent self-injurers. Journal of Consulting and Clinical Psychology, 76, 28-38. doi: 10.1037/0022-006X.76.1.28.

Nock, M.K., Joiner, T.E., Gordon, K.H., Lloyd-Richardson, E., \& Prinstein, M.J. (2006). Non-suicidal self-injury among adolescents: Diagnostic correlates and relation to suicide attempts. Psychiatry Research, 144, 65-72. doi:10.1016/j.psychres. 2006.05.010.

Patton, G.C., Coffey, C., Posterino, J.B., Carlin, J.B., \& Wolfe, R. (2001). Parental 'affectionless control' in adolescent depressive disorder. Social Psychiatry and Psychiatric Epidemiology, 36, 475-480. doi: 10.1007/s001270170011.

Paus, T., Keshavan, M., \& Giedd, J. N. (2008). Why do many psychiatric disorders emerge during adolescence?. Nature Reviews Neuroscience, 9(12), 947-957.

Preacher, K. J., \& Hayes, A. F. (2008). Asymptotic and resampling strategies for assessing and comparing indirect effects in multiple mediator models. Behavior Research Methods, 40, 879891. doi: 10.3758/BRM.40.3.879.

Rollins, B.C., \& Thomas, D.L. (1979). Parental support, power and control techniques in the socialization of children. In W.R. Burr, R. Hill, F.I. Nye, \& I.L. Reiss (Eds.). Contemporary theories about the family (pp. 317-364). London: Free Press.

Skegg, K. (2005). Self-harm. The Lancet, 366, 1471-1483. doi:10.1016/S0140-6736(05)67600-3.

Tucker, L.R., \& Lewis, C. (1973). A reliability coefficient for maximum likelihood factor analysis. Psychometrika, 38, 1-10.

Van Leeuwen, K., \&, Vermulst, A. (2004). Some psychometric properties of the Ghent parental behavior scale. European Journal of Psychological Assessment, 20, 283-298. doi: $10.1027 / 1015-5759.20 .4 .283$.

Whitlock, J., Muehlenkamp, J., Purington, A., Eckenrode, J., Barreira, J., Abrams, G. B., ... Knox, K. (2011). Non-suicidal self-injury in a college population: General trends and sex differences. 
Running title: Longitudinal study of NSSI

Journal of American College Health, 59, 691-698. doi: 10.1080/07448481.2010.529626.

Wilcox, H.C., Arria, A.M., Caldeira, K.M., Vincent, K.B., Pinchevsky, G.M., \& O'Grady, K.E. (2012). Longitudinal predictors of past-year non-suicidal self-injury and motives among college students. Psychological Medicine, 42, 717-726. doi:10.1017/S0033291711001814.

You, J., \& Leung, F. (2012). The role of depressive symptoms, family invalidation and behavioral impulsivity in the occurrence and repetition of non-suicidal self-injury in Chinese adolescents:
A 2-year follow-up study. Journal of Adolescence, 35, 389-395. doi: 10.1016/j.adolescence.2011.07.020. 

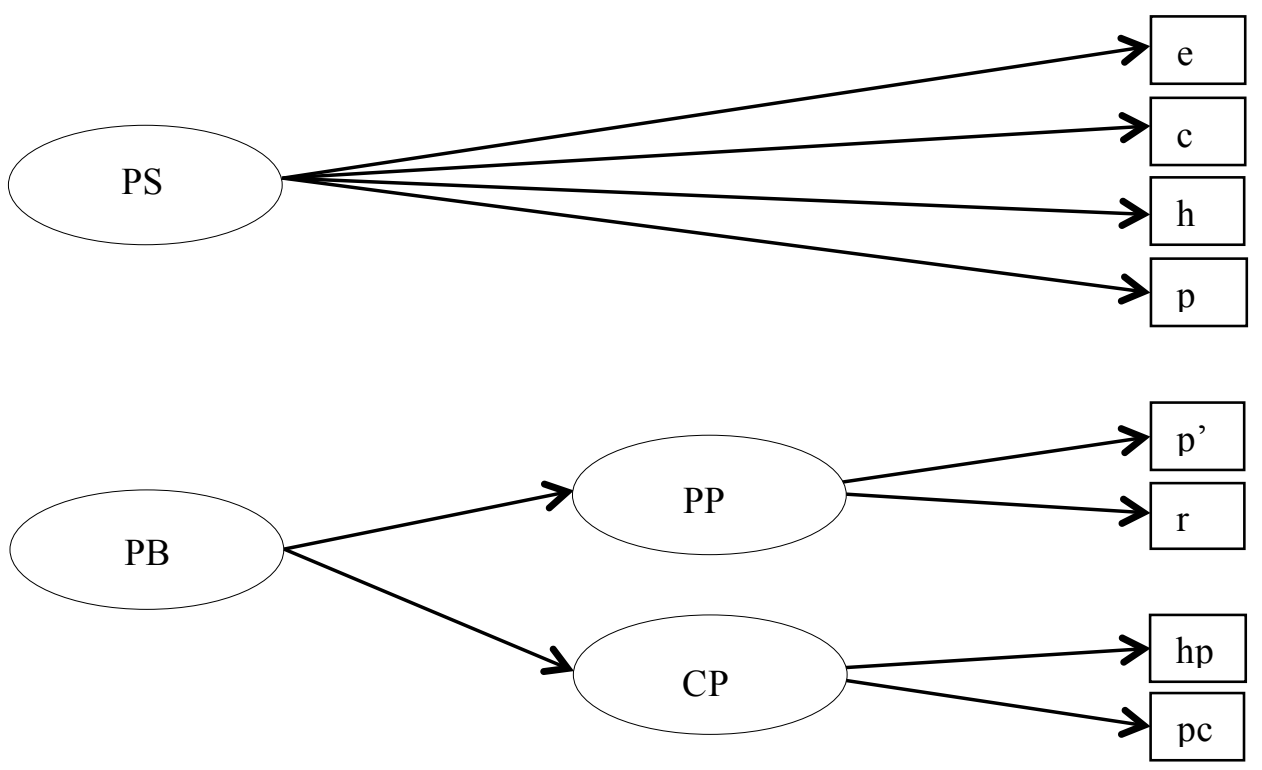

Figure 1. Final model with two latent factors "psychological distress" and "perceived parenting behaviors", with all standardized estimates significant at $p<.001(n=964)$, except for $c p, h p$ and $p c$ $(p=.24, p=.20$ and $p=.20$, respectively).

Note. $\mathrm{PS}=$ Psychological distress; $\mathrm{e}=$ emotional symptoms; $\mathrm{c}=$ conduct problems; $\mathrm{h}=$ hyperactivity/inattention; $\mathrm{p}=$ peer relationship problems; $\mathrm{PB}=$ perceived parenting behaviors; $\mathrm{PP}=$ positive parenting; $\mathrm{CP}=$ controlling parental behaviors; $\mathrm{p}^{\prime}=$ positive behaviors; $\mathrm{r}=$ rules; $\mathrm{hp}=$ harsh punishment; $\mathrm{pc}=$ psychological controlling. 


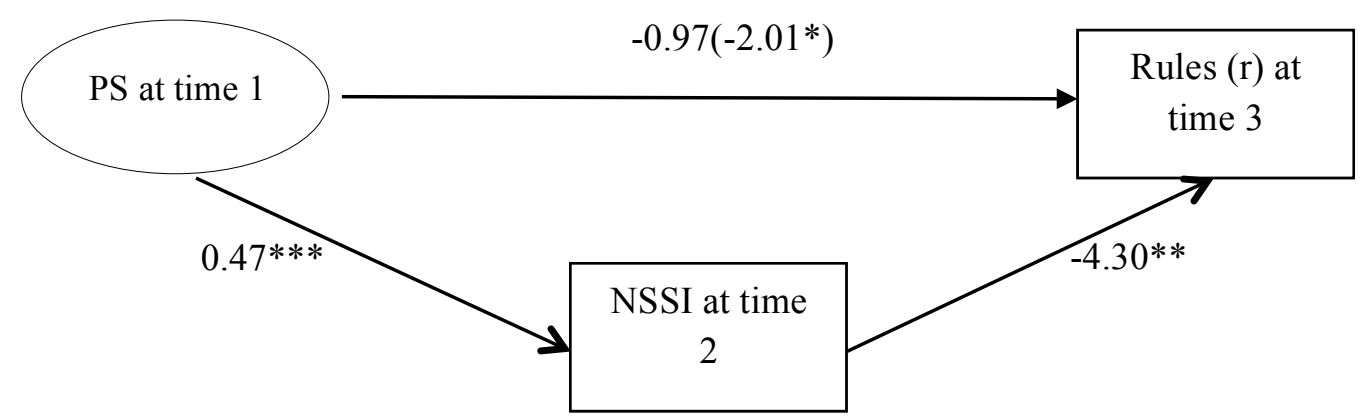

Figure 2. Regression coefficients for the mediation model with NSSI at time 2 as mediator.

Note 1. Path values are unstandardized regression coefficients. Value in parentheses are the indirect effect of psychological distress on perceived rules after the mediator (NSSI time 2) is included.

Note 2. PS = Psychological distress; NSSI = non-suicidal self-injury; $\mathrm{r}=$ rules.

${ }^{*} p<.05,{ }^{* *} p<.01,{ }^{* * *} p<.001$

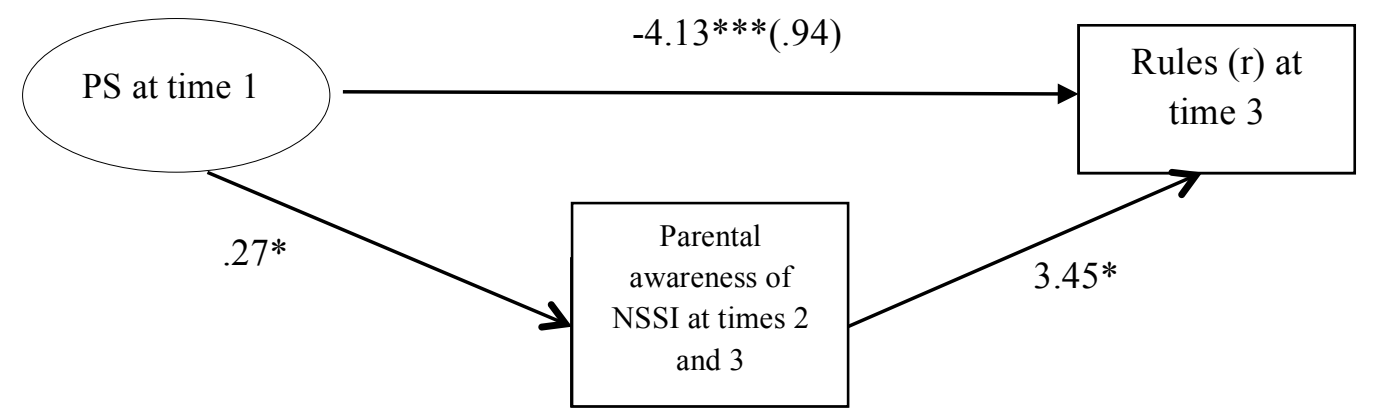

Figure 3. Regression coefficients for the mediation model with parental awareness of NSSI at time 2 and/or time 3 as the mediator.

Note 1. Path values represent unstandardized regression coefficients. Value in parentheses represents the indirect effect of psychological distress on perceived rules after the mediator (parents knowing about NSSI at time 2 or 3 ) is included.

Note 2. $\mathrm{PS}=$ Psychological distress; NSSI $=$ non-suicidal self-injury; $\mathrm{r}=$ rules.

$* p<.05, * * p<.01, * * * p<.001$ 
Running title: Longitudinal study of NSSI

Table 1

Cronbach's alphas, means, standard deviations of the latent factors on three time points.

Alpha $\quad M(\mathrm{SD})$

\begin{tabular}{lcc}
\hline PS time 1 & .78 & $24.95(13.29)$ \\
PP time 1 & .87 & $69.17(16.89)$ \\
CP time 1 & .83 & $14.63(13.84)$ \\
PS time 2 & .77 & $22.60(12.38)$ \\
PP time 2 & .89 & $72.06(17.31)$ \\
CP time 2 & .85 & $13.76(11.75)$ \\
PS time 3 & .75 & $23.03(11.88)$ \\
PP time 3 & .90 & $70.08(16.10)$ \\
CP time 3 & .85 & $14.01(11.44)$
\end{tabular}

Note. $\mathrm{PS}=$ Psychological distress; $\mathrm{pp}=$ positive parenting; $\mathrm{cp}=$ controlling parental

$* p<.05, * * p<.01, * * * p<.001$ 
Running title: Longitudinal study of NSSI

Table 2

Logistic Regression Analyses Predicting NSSI by self-reported psychological distress and parenting behaviors (support and control) and the interaction effects of support-control.

\begin{tabular}{llllll}
\hline & & \multicolumn{5}{c}{ NSSI time 2 or time 3 } \\
\hline \multirow{2}{*}{ Step 1 } & Psychiatric Disorder & $B$ & $S E$ & OR & $p$ \\
\cline { 3 - 6 } & Gender & -.09 & .48 & .92 & .85 \\
& NSSI time 1 & -.72 & .37 & .49 & $.05^{*}$ \\
Step 2 & Psychological distress & .82 & .31 & 2.26 & .15 \\
& Positive parenting & .12 & .23 & 1.12 & $.01^{* *}$ \\
& Controlling behaviors & -.13 & .35 & .82 & .71 \\
Step 3 & PP*CP (interaction) & -.02 & .13 & .98 & .86
\end{tabular}

Note. $\mathrm{PP}=$ Perceived positive parenting behaviors; $\mathrm{CP}=$ Controlling parental behaviors

${ }^{*} p<.05, * * p<.01, * * * p<.001$ 
Running title: Longitudinal study of NSSI

Table 3

Summary of mediation analyses

\begin{tabular}{|c|c|c|c|}
\hline Relationship & Standardized indirect effect & $S E$ & $p$ \\
\hline$P S \rightarrow N S S I \rightarrow r$ & -0.11 & .05 & $.03 *$ \\
\hline$P S \rightarrow N S S I \rightarrow p$ & -0.05 & .05 & .24 \\
\hline$P S \rightarrow N S S I \rightarrow h p$ & 0.01 & .06 & .88 \\
\hline$P S \rightarrow N S S I \rightarrow p c$ & -0.01 & .06 & .96 \\
\hline
\end{tabular}

Note. PS $=$ Psychological distress; NSSI $=$ non-suicidal self-injury; $\mathrm{p}=$ positive parenting; $\mathrm{r}=$ rules; $\mathrm{hp}$ $=$ harsh punishment; $\mathrm{pc}=$ psychological controlling.

$* p<.05$ 